\title{
PARTITION REGULARITY WITHOUT THE COLUMNS PROPERTY
}

\author{
BEN BARBER, NEIL HINDMAN, IMRE LEADER, AND DONA STRAUSS
}

(Communicated by Patricia L. Hersh)

\begin{abstract}
A finite or infinite matrix $A$ with rational entries is called partition regular if whenever the natural numbers are finitely coloured there is a monochromatic vector $x$ with $A x=0$. Many of the classical theorems of Ramsey Theory may naturally be interpreted as assertions that particular matrices are partition regular. In the finite case, Rado proved that a matrix is partition regular if and only it satisfies a computable condition known as the columns property. The first requirement of the columns property is that some set of columns sums to zero.

In the infinite case, much less is known. There are many examples of matrices with the columns property that are not partition regular, but until now all known examples of partition regular matrices did have the columns property. Our main aim in this paper is to show that, perhaps surprisingly, there are infinite partition regular matrices without the columns property in fact, having no set of columns summing to zero.

We also make a conjecture that if a partition regular matrix (say with integer coefficients) has bounded row sums then it must have the columns property, and prove a first step towards this.
\end{abstract}

\section{INTRODUCTION}

Let $A$ be an $u \times v$ matrix with rational entries. We say that $A$ is kernel partition regular, or simply partition regular, if for every finite colouring of the natural numbers $\mathbb{N}=\{1,2, \ldots\}$ there is a monochromatic vector $\vec{x} \in \mathbb{N}^{v}$ with $A \vec{x}=\overrightarrow{0}$. In other words, $A$ is partition regular if for every positive integer $k$, and every function $c: \mathbb{N} \rightarrow\{1, \ldots, k\}$, there is a vector $\vec{x}=\left(x_{1}, \ldots, x_{v}\right) \in \mathbb{N}^{v}$ with $c\left(x_{1}\right)=\ldots=c\left(x_{v}\right)$ such that $A \vec{x}=\overrightarrow{0}$. We may also speak of the 'system of equations $A \vec{x}=\overrightarrow{0}$ ' being partition regular.

Many of the classical results of Ramsey Theory may naturally be considered as statements about partition regularity. For example, Schur's Theorem [14, that in any finite colouring of the natural numbers we may solve $x+y=z$ in one colour class, is precisely the assertion that the $1 \times 3$ matrix $(1,1,-1)$ is partition regular. As another example, the theorem of van der Waerden [16] that, for any $m$, every finite colouring of the natural numbers contains a monochromatic arithmetic progression with $m$ terms, is (with the strengthening that we may also choose the common difference of the sequence to have the same colour) exactly the statement

Received by the editors January 6, 2014 and, in revised form, April 4, 2014.

2010 Mathematics Subject Classification. Primary 05D10.

The second author acknowledges support received from the National Science Foundation (USA) via Grant DMS-1160566. 
that the $(m-1) \times(m+1)$ matrix

$$
\left(\begin{array}{ccccccc}
1 & 1 & -1 & 0 & \ldots & 0 & 0 \\
1 & 0 & 1 & -1 & \ldots & 0 & 0 \\
1 & 0 & 0 & 1 & \ldots & 0 & 0 \\
\vdots & \vdots & \vdots & \vdots & \ddots & \vdots & \vdots \\
1 & 0 & 0 & 0 & \ldots & 1 & -1
\end{array}\right)
$$

is partition regular.

In 1933 Rado characterized those matrices that are partition regular over $\mathbb{N}$ in terms of the columns property.

Definition 1.1. Let $u, v \in \mathbb{N}$ and let $A$ be a $u \times v$ matrix with entries from $\mathbb{Q}$. Denote the columns of $A$ by $\left\langle\vec{c}_{i}\right\rangle_{i=0}^{v-1}$. The matrix $A$ satisfies the columns property if and only if there exist $m \in\{1,2, \ldots, v\}$ and a partition $\left\langle I_{t}\right\rangle_{t=0}^{m-1}$ of $\{0,1, \ldots, v-1\}$ such that

(1) $\sum_{i \in I_{0}} \vec{c}_{i}=\overrightarrow{0}$ and

(2) for each $t \in\{1,2, \ldots, m-1\}, \sum_{i \in I_{t}} \vec{c}_{i}$ is a linear combination with coefficients from $\mathbb{Q}$ of $\left\{\vec{c}_{i}: i \in \bigcup_{j=0}^{t-1} I_{j}\right\}$.

Theorem 1.2. Let $u, v \in \mathbb{N}$ and let $A$ be $a u \times v$ matrix with entries from $\mathbb{Q}$. Then $A$ is partition regular if and only if $A$ satisfies the columns property.

Proof. [13, Satz IV].

In this paper, we are concerned with partition regularity of infinite systems of homogeneous linear equations or, equivalently, with partition regularity of infinite matrices. In all cases we shall assume that the equations we are dealing with have finitely many terms; that is, each row of the matrix of coefficients has all but finitely many entries equal to 0 . The definition of partition regularity for a matrix (or a system of linear equations) is verbatim the same as for finite matrices or systems of equations.

The first example of a (non-trivial) infinite partition regular system of equations was constructed in 1974 [5], proving a conjecture of Graham and Rothschild: in any finite colouring of the natural numbers there is a sequence $x_{1}, x_{2}, \ldots$ of natural numbers such that the set

$$
F S\left(x_{1}, x_{2}, \ldots\right)=\left\{\sum_{i \in I} x_{i}: I \subseteq \mathbb{N}, I \text { finite and non-empty }\right\}
$$

is monochromatic. This is also known as the Finite Sums Theorem. (It is worth remarking that the finite analogue of this, known as Folkman's Theorem, stating that, for any $m$, in any finite colouring of the natural numbers we may find $x_{1}, \ldots, x_{m}$ with $F S\left(x_{1}, \ldots, x_{m}\right)$ monochromatic, follows easily from Rado's Theorem.) Since then, several other infinite partition systems have been found - for example, the Milliken-Taylor Theorem [12, 15 and several systems in 6]. See 77 for general background on this.

We take $\omega=\mathbb{N} \cup\{0\}$. The columns property has an obvious extension to infinite matrices.

Definition 1.3. Let $u, v \in \mathbb{N} \cup\{\omega\}$ and let $A$ be a $u \times v$ matrix with entries from $\mathbb{Q}$. Denote the columns of $A$ by $\left\langle\vec{c}_{i}\right\rangle_{i<v}$. The matrix $A$ satisfies the columns property if and only if there exists a partition $\left\langle I_{\sigma}\right\rangle_{\sigma<\mu}$ of $v$, where $\mu \in \mathbb{N} \cup\{\omega\}$, such that 
(1) $\sum_{i \in I_{0}} \vec{c}_{i}=\overrightarrow{0}$ and

(2) for each $t \in \mu \backslash\{0\}, \sum_{i \in I_{t}} \vec{c}_{i}$ is a linear combination with coefficients from $\mathbb{Q}$ of $\left\{\vec{c}_{i}: i \in \bigcup_{j<t} I_{j}\right\}$.

We stress that the sums in this definition are not required to be finite. Note that infinite sums of columns do always make sense, because our matrices have only finitely many non-zero entries in each row. It is also worth remarking that if one insisted on finite sums then nothing would work: even the matrix corresponding to the Finite Sums Theorem needs infinite sums for the columns property.

It is easy to see that, for infinite matrices, the columns property is not sufficient for partition regularity. Consider for example the system of equations $x_{n}-x_{n+1}=$ $y_{n}, n \in \omega$. As a matrix equation this corresponds to

$$
\left(\begin{array}{cccccccc}
1 & -1 & -1 & 0 & 0 & 0 & 0 & \cdots \\
0 & 0 & 1 & -1 & -1 & 0 & 0 & \cdots \\
0 & 0 & 0 & 0 & 1 & -1 & -1 & \ldots \\
\vdots & \vdots & \vdots & \vdots & \vdots & \vdots & \vdots & \ddots
\end{array}\right)\left(\begin{array}{c}
x_{0} \\
y_{0} \\
x_{1} \\
y_{1} \\
\vdots
\end{array}\right)=\overrightarrow{0}
$$

The system of equations is not partition regular over $\mathbb{N}$ for the trivial reason that it has no solutions there at all; any solution must have $x_{n}>x_{n+1}$ for each $n$. To see that the matrix satisfies the columns property, let $I_{0}=\{0,2,4,6, \ldots\}$ and $I_{1}=\{1,3,5, \ldots\}$. Then $\sum_{i \in I_{0}} \vec{c}_{i}=\overrightarrow{0}$ and $\sum_{i \in I_{1}} \vec{c}_{i}=\sum_{t=1}^{\infty} t \vec{c}_{2 t}$.

In the converse direction, it has been the case until now that all known partition regular systems of equations did have the columns property. However, we shall show in Section 2 of this paper that there is a system of linear equations which is partition regular over $\mathbb{N}$ but for which the coefficient matrix has no set of columns summing to zero. We mention in passing that the entries and the columns of this matrix will be 'nicely behaved': all entries are from the set $\{-1,0,1,2\}$ and each column of the coefficient matrix has at most three nonzero entries.

The example mentioned in the previous paragraph has the property that the row sums of the coefficient matrix are unbounded. We also show in Section 2 that if $A$ is a matrix which is partition regular over $\mathbb{N}$ and the sums of the absolute values of the entries in any row are bounded, then there must be some set of columns that sum to zero. We do not know whether such a matrix must satisfy the full columns property.

The proof that the system mentioned above is partition regular over $\mathbb{N}$ depends on the following lemma, which is based on results in 2]. The lemma refers to central sets. From the point of view of the partition regularity of our system of equations, central sets have two key properties.

- Whenever $\mathbb{N}$ is finitely coloured, one of the colour classes must be central.

- Any finite partition regular system of homogeneous linear equations has solutions in any central set (see [4, Theorem 8.22]). (The corresponding statement is not true for infinite systems.)

In the statement of this lemma, $m \cdot \mathbb{Z}=\{m \cdot n: n \in \mathbb{Z}\}$ while $k C=C+C+\cdots+C$ ( $k$ times).

Lemma 1.4. Let $C$ be a central subset of $\mathbb{N}$. There exist $m$ and $K$ in $\mathbb{N}$ such that if $k \geq K$, then $m \cdot \mathbb{Z} \subseteq C-k C$. 
Section 3 consists of a proof of (a generalisation of) Lemma 1.4, and also a strengthening of the main result of Section 2 (namely Theorem 2.1). The proofs depend on the algebraic structure of the Stone-Cech compactification $\beta \mathbb{N}$ of the discrete space $\mathbb{N}$. We give a brief introduction to that structure in that section. We mention that the point of Lemma 1.4, and the point of using central sets, is that then the proof of Theorem 2.1 is clean and direct.

\section{Partition REgularity DOES NOt IMPly THE COLUMNS PROPERTY}

In this section we consider the following system of equations:

$$
2 x_{n}+x_{2^{n}}+x_{2^{n}+1}+\cdots+x_{2^{n+1}-1}=y_{n}, \quad n \in \omega .
$$

The simplest way to represent the matrix of coefficients is as an $\omega \times(\omega+\omega)$ matrix. That is, we let $A$ be the $\omega \times \omega$ matrix such that, for $i, j \in \omega$,

$$
a_{i, j}= \begin{cases}2 & \text { if } j=i \\ 1 & \text { if } 2^{i} \leq j<2^{i+1} \\ 0 & \text { otherwise }\end{cases}
$$

so that

$$
A=\left(\begin{array}{cccccccccc}
2 & 1 & 0 & 0 & 0 & 0 & 0 & 0 & 0 & \ldots \\
0 & 2 & 1 & 1 & 0 & 0 & 0 & 0 & 0 & \ldots \\
0 & 0 & 2 & 0 & 1 & 1 & 1 & 1 & 0 & \ldots \\
\vdots & \vdots & \vdots & \vdots & \vdots & \vdots & \vdots & \vdots & \vdots & \ddots
\end{array}\right)
$$

Letting $I$ be the $\omega \times \omega$ identity matrix, we then have that the matrix of coefficients for our system of equations is $(A-I)$. It is immediate that there is no non-empty set of columns of this matrix summing to $\overrightarrow{0}$.

Theorem 2.1. For any central subset $C$ of $\mathbb{N}$, there exist $\vec{x}$ and $\vec{y}$ in $C^{\omega}$ such that $\left(\begin{array}{ll}A & -I\end{array}\right)\left(\begin{array}{l}\vec{x} \\ \vec{y}\end{array}\right)=\overrightarrow{0}$. In particular the system of equations

$$
2 x_{n}+x_{2^{n}}+x_{2^{n}+1}+\cdots+x_{2^{n+1}-1}=y_{n}, \quad n \in \omega,
$$

is partition regular over $\mathbb{N}$.

Proof. Pick $m$ and $K$ as guaranteed for $C$ by Lemma 1.4. Pick $M \in \mathbb{N}$ such that $2^{M+1} \geq m-2+K$. Let $P$ be the $(M+1) \times 2^{M+1}$ matrix consisting of rows $0,1, \ldots, M$ and columns $0,1, \ldots, 2^{M+1}-1$ of $A$ and let $I_{M+1}$ be the $(M+1) \times(M+1)$ identity matrix. Then $\left(\begin{array}{ll}P & -I_{M+1}\end{array}\right)$ satisfies the columns property. Since, as we remarked earlier, any finite partition regular system of homogeneous linear equations has solutions in any central set, we may choose $\vec{x}=\left\langle x_{i}\right\rangle_{i=0}^{2^{M+1}-1}$ and $\vec{y}=\left\langle y_{i}\right\rangle_{i=0}^{M}$ in $C$ such that $\left(\begin{array}{ll}P & -I_{M+1}\end{array}\right)\left(\begin{array}{l}\vec{x} \\ \vec{y}\end{array}\right)=\overrightarrow{0}$. That is, for each $n \in\{0,1, \ldots, M\}$, the equation $2 x_{n}+x_{2^{n}}+x_{2^{n}+1}+\cdots+x_{2^{n+1}-1}=y_{n}$ holds.

Let $r \geq M$ and assume that we have chosen $\left\langle x_{i}\right\rangle_{i=0}^{2^{r+1}-1}$ and $\left\langle y_{i}\right\rangle_{i=0}^{r}$ in $C$ such that for each $n \in\{0,1, \ldots, r\}$, the equation $2 x_{n}+x_{2^{n}}+x_{2^{n}+1}+\cdots+x_{2^{n+1}-1}=y_{n}$ holds. For $2^{r+1} \leq t \leq 2^{r+1}+m-3$, let $x_{t}=x_{r+1}$. Let $k=2^{r+1}-m+2$. Then $k \geq K$ and

$$
2 x_{r+1}+x_{2^{r+1}}+x_{2^{r+1}+1}+\cdots+x_{2^{r+1}+m-3}=m \cdot x_{r+1} \in m \cdot \mathbb{Z},
$$


so by Lemma 1.4 we may pick $y_{r+1} \in C$ and pick $x_{t} \in C$ for $2^{r+1}+m-2 \leq t \leq$ $2^{r+2}-1$ such that $2 x_{r+1}+x_{2^{r+1}}+x_{2^{r+1}+1}+\cdots+x_{2^{r+2}-1}=y_{r+1}$.

We shall see in Corollary 3.8 that in fact, one may choose $\vec{x}$ and $\vec{y}$ in $C^{\omega}$ such that $\left(\begin{array}{ll}A & -I\end{array}\right)\left(\begin{array}{l}\vec{x} \\ \vec{y}\end{array}\right)=\overrightarrow{0}$ and all entries of $\left(\begin{array}{l}\vec{x} \\ \vec{y}\end{array}\right)$ are distinct.

The matrix of Theorem 2.1 has unbounded row sums. This motivates the following. Let us say that an $\omega \times \omega$ matrix $A$ with entries from $\mathbb{Z}$ has bounded row sums if $\left\{\sum_{j<\omega}\left|a_{i, j}\right|: i<\omega\right\}$ is bounded.

We now see that if $A$ is a matrix which is partition regular and has bounded row sums then in fact some non-empty set of columns must sum to zero. In fact, this is pretty much a direct copy of Rado's original proof in the finite case.

Theorem 2.2. Let $A$ be an $\omega \times \omega$ matrix with entries from $\mathbb{Z}$ having bounded row sums. If $A$ is $K P R / \mathbb{N}$ and $\vec{c}_{i}$ denotes column $i$ of $A$, then there is some $J \subseteq \omega$ such that $\sum_{i \in J} \vec{c}_{i}=\overrightarrow{0}$.

Proof. Pick a prime $q$ such that $\sum_{j<\omega}\left|a_{i, j}\right|<q$ for each $i<\omega$. Given $x \in \mathbb{N}$, pick $b(x) \in\{1,2, \ldots, q-1\}, l(x) \in \omega$ and $a(x) \in \omega$ such that $x=b(x) q^{l(x)}+a(x) q^{l(x)+1}$. (Thus $b(x)$ is the rightmost nonzero digit in the base $q$ expansion of $x$ and $l(x)$ is the position of that digit.) For $b \in\{1,2, \ldots, q-1\}$, let $C_{b}=\{x \in \mathbb{N}: b(x)=b\}$. Pick $b \in\{1,2, \ldots, q-1\}$ and $\vec{x} \in C_{b}^{\omega}$ such that $A \vec{x}=\overrightarrow{0}$. Let $d=\min \left\{l\left(x_{i}\right): i<\omega\right\}$ and let $J=\left\{i<\omega: l\left(x_{i}\right)=d\right\}$. We shall show that $\sum_{j \in I} \vec{c}_{j}=\overrightarrow{0}$.

For $j \in J$, let $e_{j}=a\left(x_{j}\right)$ so that $x_{j}=b q^{d}+e_{j} q^{d+1}$. For $j \in \omega \backslash J, l\left(x_{j}\right)>d$ so pick $e_{j} \in \mathbb{N}$ such that $x_{j}=e_{j} q^{d+1}$. Suppose that $\sum_{j \in J} \vec{c}_{j} \neq \overrightarrow{0}$ and pick some $i<\omega$ such that $\sum_{j \in J} a_{i, j} \neq 0$. Then $0=\sum_{j \in J} b q^{d} a_{i, j}+\sum_{j<\omega} e_{j} q^{d+1} a_{i, j}$ so $q$ divides $\sum_{j \in J} b a_{i, j}$ so $q$ divides $\sum_{j \in J} a_{i, j}$ while $q>\sum_{j \in J}\left|a_{i, j}\right|$, a contradiction.

Remark 2.3. A matrix $A$ which satisfies the weaker condition that $\left\{\left|\sum_{j<\omega} a_{i, j}\right|\right.$ : $i<\omega\}$ is bounded could be partition regular and have no non-empty set of columns summing to zero. If

$$
A=\left(\begin{array}{ccccccccc}
-2 & 1 & 0 & 0 & 0 & 0 & 0 & 0 & \ldots \\
0 & -3 & 1 & 1 & 0 & 0 & 0 & 0 & \ldots \\
0 & 0 & -4 & 0 & 1 & 1 & 1 & 0 & \ldots \\
\vdots & \vdots & \vdots & \vdots & \vdots & \vdots & \vdots & \vdots & \ddots
\end{array}\right)
$$

then $(A-I)$ is partition regular; the proof is essentially the same as that of Theorem 2.1. Clearly, there is no non-empty set of columns of $\left(\begin{array}{ll}A-I & -1) \text { whose sum }\end{array}\right.$ is zero.

Very frustratingly, we have been unable to extend Theorem 2.2 to the following.

Conjecture 2.4. Let $A$ be an $\omega \times \omega$ matrix with entries from $\mathbb{Z}$, and assume that $A$ is partition regular. If $A$ has bounded row sums, then $A$ has the columns property.

If this conjecture is false, might at least a weaker statement be true, that $A$ must satisfy the natural 'transfinite' version of the columns property?

Definition 2.5. Let $u, v \in \mathbb{N} \cup\{\omega\}$ and let $A$ be a $u \times v$ matrix with entries from $\mathbb{Q}$. Denote the columns of $A$ by $\left\langle\vec{c}_{i}\right\rangle_{i<v}$. The matrix $A$ satisfies the transfinite columns 
property if and only if there exist a countable ordinal $\mu$ and a partition $\left\langle I_{\sigma}\right\rangle_{\sigma<\mu}$ of $v$ such that

(1) $\sum_{i \in I_{0}} \vec{c}_{i}=\overrightarrow{0}$ and

(2) for each $t \in \mu \backslash\{0\}, \sum_{i \in I_{t}} \vec{c}_{i}$ is a linear combination with coefficients from $\mathbb{Q}$ of $\left\{\vec{c}_{i}: i \in \bigcup_{j<t} I_{j}\right\}$.

We remark that, for any given countable ordinal $\mu$, it is a straightforward exercise to write down a matrix that has the transfinite columns property with the partition being indexed by $\mu$, but without any partition having a smaller indexing ordinal.

Our weaker version of Conjecture 2.4 would then be:

Conjecture 2.6. Let $A$ be an $\omega \times \omega$ matrix with entries from $\mathbb{Z}$ and assume that $A$ is partition regular. If $A$ has bounded row sums, then $A$ has the transfinite columns property.

We saw in the Introduction that the system of equations $x_{n}-x_{n+1}=y_{n}$ satisfies the columns property, but is not partition regular over $\mathbb{N}$. However, as shown at the end of Section 3 of [7, it is partition regular over $\mathbb{R}$ - meaning that whenever $\mathbb{R} \backslash\{0\}$ is finitely coloured there is a monochromatic solution to that system of equations. (It was also shown there that it is not partition regular over $\mathbb{Q}$.) This leaves open the possibility that perhaps a sufficiently well-behaved system of equations which satisfies the columns property must at least be partition regular over $\mathbb{R}$. To end this section, we show that this seems not to be the case. The system $x_{n}+2 x_{n+1}=y_{n}$ is about as well behaved as one can wish. The sum of absolute values of entries of each row of the coefficient matrix is 4 and all columns sum to $1,-1$, or 3 . The matrix equation is

$$
\left(\begin{array}{ccccccccc}
1 & -1 & 2 & 0 & 0 & 0 & 0 & 0 & \ldots \\
0 & 0 & 1 & -1 & 2 & 0 & 0 & 0 & \cdots \\
0 & 0 & 0 & 0 & 1 & -1 & 2 & 0 & \cdots \\
\vdots & \vdots & \vdots & \vdots & \vdots & \vdots & \vdots & \vdots & \ddots
\end{array}\right)\left(\begin{array}{c}
x_{0} \\
y_{0} \\
x_{1} \\
y_{1} \\
\vdots
\end{array}\right)=\overrightarrow{0}
$$

The matrix of coefficients satisfies the columns property with $I_{i}=\{2 i, 2 i+1\}$ for each $i<\omega$.

Theorem 2.7. The system of equations $x_{n}+2 x_{n+1}=y_{n}$ is not partition regular over $\mathbb{R}$.

Proof. We show first that the system is not partition regular over $\mathbb{Q}$, for which it suffices to show that it is not partition regular over $\mathbb{Q}^{+}=\{x \in \mathbb{Q}: x>0\}$. We begin by defining $\tau: \mathbb{Q}^{+} \rightarrow\{0,1,2\}$ by $\tau(x)=i$ if and only if $\left\lfloor\log _{2}(x)\right\rfloor \equiv i$ $(\bmod 3)$. Note that if $\tau(x)=\tau(y)$ and $y \geq 2 x$, then $y>4 x$. (To see this, let $k=\left\lfloor\log _{2}(x)\right\rfloor$ and $m=\left\lfloor\log _{2}(y)\right\rfloor$. Then $2^{m+1}>y \geq 2 x \geq 2^{k+1}$, so $m>k$ and therefore, $m \geq k+3$. Then $y \geq 2^{m} \geq 2^{k+3}>4 x$.)

As is relatively well known, and at any rate easy to verify, every rational $x \in(0,1)$ has a unique expansion of the form

$$
x=\sum_{t=2}^{m(x)} \frac{a(x, t)}{t !},
$$

where each $a(x, t) \in\{0,1, \ldots, t-1\}$ and $a(x, m(x))>0$. If $t>m(x)$, let $a(x, t)=0$. For each $t \geq 2$, choose $\nu_{t}:\{1,2, \ldots, t-1\} \rightarrow\{0,1,2\}$ such that, if $i, j \in\{1,2, \ldots, t-1\}$ 
and $j \equiv 2 i \quad(\bmod t)$, then $\nu_{t}(i) \neq \nu_{t}(j)$. (To see that such a colouring exists, let $G$ be the digraph on vertex set $\{1,2, \ldots, t-1\}$ with an edge from $i$ to $j$ whenever $j \equiv 2 i$ $(\bmod t)$. Then every edge of $G$ is in at most one cycle, so $G$ can be 3 -coloured.)

Now define a finite colouring $\varphi$ of $\mathbb{Q}^{+}$so that for $x, y \in \mathbb{Q}^{+}, \varphi(x)=\varphi(y)$ if and only if

(1) $\tau(x)=\tau(y)$;

(2) $x<1$ if and only if $y<1$; and

(3) if $x<1$ and $y<1$, then

(a) $m(x) \equiv m(y) \quad(\bmod 3)$ and

(b) if $m(x)=m(y)$, then $\nu_{m(x)}(a(x, m(x)))=\nu_{m(y)}(a(y, m(y)))$.

Now suppose we have a system $x_{n}+2 x_{n+1}=y_{n}$ for $n<\omega$ such that $\left\{x_{n}\right.$ : $n<\omega\} \cup\left\{y_{n}: n<\omega\right\}$ is monochromatic with respect to $\varphi$. Given $n$, we have that $y_{n}>2 x_{n+1}$ so, since $\tau\left(y_{n}\right)=\tau\left(x_{n+1}\right)$, we have $y_{n}>4 x_{n+1}$. But then $x_{n}=y_{n}-2 x_{n+1}>2 x_{n+1}$. Therefore, the sequence $\left\langle x_{n}\right\rangle_{n=0}^{\infty}$ is eventually less than 1 and therefore,

$$
\left\{x_{n}: n<\omega\right\} \cup\left\{y_{n}: n<\omega\right\} \subseteq(0,1) .
$$

Given any $k,\{x \in(0,1): m(x) \leq k\}$ is finite and therefore, there is some $n$ such that $m\left(x_{n+1}\right)>m\left(x_{n}\right)$ and consequently $m\left(x_{n+1}\right) \geq m\left(x_{n}\right)+3$. Let $m=m\left(x_{n+1}\right)$, let $(d, c, b)=\left(a\left(x_{n+1}, m-2\right), a\left(x_{n+1}, m-1\right), a\left(x_{n+1}, m\right)\right)$, and let $\left(d^{\prime}, c^{\prime}, b^{\prime}\right)=$ $\left(a\left(y_{n}, m-2\right), a\left(y_{n}, m-1\right), a\left(y_{n}, m\right)\right)$. Now $b^{\prime} \equiv 2 b \quad(\bmod m)$ so, since $\varphi\left(y_{n}\right)=$ $\varphi\left(x_{n+1}\right)$ we must have that $b^{\prime}=0$ and therefore, $m\left(y_{n}\right)<m\left(x_{n+1}\right)=m$. Thus $m\left(y_{n}\right) \leq m-3$ and so $d^{\prime}=c^{\prime}=0$. Since $0 \equiv 2 b \quad(\bmod m)$ we must have that $m=2 b$. Since there is a carry out of position $m$ when $2 x_{n+1}$ is computed, $0=c^{\prime} \equiv 2 c+1 \quad(\bmod m-1)$ and so there is a carry out of position $m-1$. But then $0=d^{\prime} \equiv 2 d+1(\bmod m-2)$, which is impossible since $m-2$ is even.

Thus we have established that our system is not partition regular over $\mathbb{Q}^{+}$. Extend the colouring $\varphi$ to a colouring $\varphi^{\prime}$ of $\mathbb{Q} \backslash\{0\}$ with respect to which there is no monochromatic system (e.g., by reflecting to $\{x \in \mathbb{Q}: x<0\}$ using a new set of colours).

Now pick a Hamel basis $B$ for $\mathbb{R}$ over $\mathbb{Q}$ and well-order $B$. For $x \in \mathbb{R} \backslash\{0\}$ let $\operatorname{supp}(x)$ be the finite non-empty subset of $B$ such that $x=\sum_{b \in \operatorname{supp}(x)} \alpha(x, b) b$ and each $\alpha(x, b) \in \mathbb{Q} \backslash\{0\}$ and let $b(x)=\max \operatorname{supp}(x)$.

Now let $\psi: \mathbb{Q} \backslash\{0\} \rightarrow\{0,1\}$ such that for all $x \in \mathbb{Q} \backslash\{0\}, \psi(2 x) \neq \psi(x)$. (For example, colour by the parity of the number of factors of 2 in the numerator or denominator of $x$.) Now define a finite colouring $\gamma$ of $\mathbb{R} \backslash\{0\}$ so that $\gamma(x)=\gamma(y)$ if and only if

(1) $\alpha(x, b(x))>0$ if and only if $\alpha(y, b(y))>0$;

(2) $\psi(\alpha(x, b(x)))=\psi(\alpha(y, b(y)))$; and

(3) $\varphi^{\prime}(\alpha(x, b(x)))=\varphi^{\prime}(\alpha(y, b(y)))$.

Now suppose we have a system $x_{n}+2 x_{n+1}=y_{n}$ in $\mathbb{R}$ for $n<\omega$ such that $\left\{x_{n}: n<\omega\right\} \cup\left\{y_{n}: n<\omega\right\}$ is monochromatic with respect to $\gamma$. If for any $n$ we have $b\left(x_{n+1}\right)>b\left(x_{n}\right)$, then we get $b\left(y_{n}\right)=b\left(x_{n+1}\right)$ and $\alpha\left(y_{n}, b\left(y_{n}\right)\right)=$ $2 \alpha\left(x_{n+1}, b\left(x_{n+1}\right)\right)$, contradicting requirement (2). Therefore, for each $n, b\left(x_{n+1}\right) \leq$ $b\left(x_{n}\right)$. Since there are no infinite strictly decreasing sequences in $B$, there are some $b \in B$ and $k<\omega$ such that for all $n \geq k, b\left(x_{n}\right)=b$. Note that by requirement (1), for all such $n, b\left(y_{n}\right)=b$. Now let for each $n<\omega, x_{n}^{\prime}=\alpha\left(x_{k+n}, b\right)$ and 
$y_{n}^{\prime}=\alpha\left(y_{k+n}, b\right)$. Then $\left\{x_{n}^{\prime}: n<\omega\right\} \cup\left\{y_{n}^{\prime}: n<\omega\right\}$ is monochromatic with respect to $\varphi^{\prime}$ and for each $n, x_{n}+2 x_{n+1}=y_{n}$, a contradiction.

\section{Proof of Lemma 1.4}

In this section we will provide a proof of a generalisation of Lemma 1.4. We also prove a strengthening of Theorem 2.1. These results utilise the algebraic structure of the Stone-Cech compactification $\beta S$ of a discrete semigroup $S$. We shall provide a brief introduction to this structure now. For proofs of the assertions made here, see the first four chapters of [11. We shall be concerned here almost exclusively with commutative semigroups, so we will denote the operation of $S$ by + . We mention here that the reader who is not interested in the results for general groups or semigroups can skip Lemma 3.1 and its proof and rejoin the text at Lemma 3.3. taking $G$ to be $\mathbb{Z}$ and $d$ to be the usual density for subsets of $\mathbb{Z}$.

We take the points of $\beta S$ to be the ultrafilters on $S$, the principal ultrafilters being identified with the points of $S$, whereby we pretend that $S \subseteq \beta S$. Given a subset $A$ of $S, \bar{A}=\{p \in \beta S: A \in p\}$ is the closure of $A$ in $\beta S$ and $\{\bar{A}: A \subseteq S\}$ is a basis for the open sets of $\beta S$ as well as a basis for the closed sets of $\beta S$.

The operation on $S$ extends to an operation on $\beta S$, also denoted by + , with the property that for each $p \in \beta S$, the function $\rho_{p}: \beta S \rightarrow \beta S$ is continuous and for each $x \in S$, the function $\lambda_{x}: \beta S \rightarrow \beta S$ is continuous where, for $q \in \beta S, \rho_{p}(q)=q+p$ and $\lambda_{x}(q)=x+q$. (The reader should be cautioned that $(\beta S,+)$ is almost certain to not be commutative; the centre of $(\beta \mathbb{N},+)$ is $\mathbb{N}$.) Given $A \subseteq S$ and $p, q \in \beta S, A \in p+q$ if and only if $\{x \in S:-x+A \in q\} \in p$, where $-x+A=\{y \in S: x+y \in A\}$.

Thus $(\beta S,+)$ is a compact Hausdorff right topological semigroup. As with any such object, there exist idempotents in $\beta S$. Also, $\beta S$ has a smallest two-sided ideal, $K(\beta S)$, which is the union of all of the minimal right ideals of $\beta S$ as well as the union of all of the minimal left ideals of $\beta S$. The intersection of any minimal right ideal with any minimal left ideal is a group and any two such groups are isomorphic. In particular, there are idempotents in $K(\beta S)$. A subset $C$ of $S$ is said to be central if and only if it is a member of some idempotent in $K(\beta S)$. (Such idempotents are said to be minimal.) Since whenever the union of finitely many sets is a member of an ultrafilter, one of the sets must itself be a member of the ultrafilter, one has immediately that whenever $S$ is finitely coloured, at least one colour class must be central. A subset $A$ of $S$ is said to be central* if and only if it has non-empty intersection with any central set, equivalently if and only if it is a member of every minimal idempotent.

Given a set $X$, let $\mathcal{P}_{f}(X)$ be the set of finite non-empty subsets of $X$. A subset $A$ of $S$ is syndetic if and only if there is some $L \in \mathcal{P}_{f}(S)$ such that $S=\bigcup_{t \in L}(-t+A)$. A subset $A$ of $S$ is piecewise syndetic if and only if

$$
\left(\exists G \in \mathcal{P}_{f}(S)\right)\left(\forall F \in \mathcal{P}_{f}(S)\right)(\exists x \in S)\left(F+x \subseteq \bigcup_{t \in G}(-t+A)\right) .
$$

It is then a fact that $A$ is piecewise syndetic if and only if $\bar{A} \cap K(\beta S) \neq \emptyset$. In particular, every central set is piecewise syndetic.

Lemma 3.1. Let $(S,+)$ be a commutative cancellative semigroup. There is a function $d: \mathcal{P}(S) \rightarrow[0,1]$ with the following properties.

(1) $d(S)=1$.

(2) For each piecewise syndetic subset $A$ of $S, d(A)>0$.

(3) For $B, C \subseteq S, d(B \cup C) \leq d(B)+d(C)$. 
(4) For $a \in S$ and $B \subseteq S, d(B)=d(a+B)=d(-a+B)$.

(5) If $B \subseteq S, n \in \mathbb{N}$, and $x_{1}, x_{2}, \ldots, x_{n} \in S$ such that $\left(x_{i}+B\right) \cap\left(x_{j}+B\right)=\emptyset$ if $i \neq j$, then $d\left(\bigcup_{i=1}^{n}\left(x_{i}+B\right)\right)=n d(B)$.

Proof. There are at least two ways to see this. One way is to use the following very general result, whose proof can be found in [3].

Lemma 3.2. Let $S$ be a left amenable semigroup and let $A$ be a piecewise syndetic subset of $S$. Then a probability measure $\nu$ can be defined on $\beta S$ with the following properties:

(a) $\nu\left(s^{-1} B\right)=\nu(B)$ for every Borel subset $B$ of $\beta S$ and every $s \in S$ and

(b) $\nu(\bar{A})>0$.

If, in addition $S$ is cancellative, then:

(c) $\nu(s B)=\nu(B)$ for every Borel subset $B$ of $\beta S$ and every $s \in S$.

Using this lemma, one then defines $d(B)=\sup \{\nu(\bar{B}): \nu$ is a probability measure on $\beta S$ satisfying (a) and (c) of Lemma 3.2$\}$.

An alternative, mostly elementary, method is to use the fact proved by Argabright and Wilde in [1, Theorem 4] that any commutative semigroup satisfies the Strong Følner Condition:

$$
\left(\forall H \in \mathcal{P}_{f}(S)\right)(\forall \epsilon>0)\left(\exists K \in \mathcal{P}_{f}(S)\right)(\forall s \in H)(|K \triangle(s+K)|<\epsilon \cdot|K|) .
$$

(For an entirely elementary proof of this fact see [9, Section 7].)

If $S$ satisfies the Strong Følner Condition and $A \subseteq S$, then the Følner density of $A$ is

$$
\begin{gathered}
d(A)=\sup \left\{\alpha:\left(\forall H \in \mathcal{P}_{f}(S)\right)(\forall \epsilon>0)\left(\exists K \in \mathcal{P}_{f}(S)\right)(|A \cap K| \geq \alpha \cdot|K|)\right. \text { and } \\
(\forall s \in H)(|K \triangle(s+K)|<\epsilon \cdot|K|))\} .
\end{gathered}
$$

Conclusions (1) and (3) are routine to verify for Følner density, and conclusion (4) is established in [8, Theorem 4.17]. Define

$$
\mathbf{D}=\{p \in \beta S:(\forall A \in p)(d(A)>0)\} .
$$

To verify (2), let $A$ be a piecewise syndetic subset of $S$. By [10, Theorems 2.12, 2.14, and 5.9], $\mathbf{D}$ is a two-sided ideal of $\beta S$. (Alternatively, it is a routine elementary argument to establish directly that $\mathbf{D}$ is a two-sided ideal of $\beta S$.) Since $\mathbf{D}$ is a two-sided ideal of $\beta S, K(\beta S) \subseteq \mathbf{D}$. Since $A$ is piecewise syndetic, $\bar{A} \cap K(\beta S) \neq \emptyset$ and therefore, $\bar{A} \cap \mathbf{D} \neq \emptyset$. Therefore $d(A)>0$.

Finally, it is routine to establish that conclusion (5) holds. To emphasize how routine it is, we present the full proof now. By conclusions (3) and (4), $d\left(\bigcup_{i=1}^{n}\left(x_{i}+B\right)\right) \leq \sum_{i=1}^{n} d\left(x_{i}+B\right)=n d(B)$. Let $a=d(B)$. We complete the proof by showing that if $\alpha<a$, then $d\left(\bigcup_{i=1}^{n}\left(x_{i}+B\right)\right) \geq n \alpha$.

Let $\alpha<a$ be given and let $\gamma=(a-\alpha) / 2$. To see that $d\left(\bigcup_{i=1}^{n}\left(x_{i}+B\right)\right) \geq n \alpha$, let $H \in \mathcal{P}_{f}(S)$ and $\epsilon>0$ be given. Let $H^{\prime}=H \cup\left\{x_{1}, x_{2}, \ldots, x_{n}\right\}$ and let $\epsilon^{\prime}=$ $\min \{\epsilon, \gamma\}$. Since $d(B)>\alpha+\gamma$, pick $K \in \mathcal{P}_{f}(S)$ such that $|B \cap K| \geq(\alpha+\gamma)|K|$ and $\left(\forall s \in H^{\prime}\right)\left(|K \triangle(s+K)|<\epsilon^{\prime}|K|\right)$. Then $(\forall s \in H)(|K \triangle(s+K)|<\epsilon|K|)$ so it suffices to show that $\left|\left(\bigcup_{i=1}^{n}\left(x_{i}+B\right)\right) \cap K\right| \geq n \alpha|K|$. By the disjointness assumption, $\left|\left(\bigcup_{i=1}^{n}\left(x_{i}+B\right)\right) \cap K\right|=\sum_{i=1}^{n}\left|\left(x_{i}+B\right) \cap K\right|$ so it suffices to let $i \in\{1,2, \ldots, n\}$ and show that $\left|\left(x_{i}+B\right) \cap K\right| \geq \alpha|K|$. 
So let $i \in\{1,2, \ldots, n\}$ be given. Since $x_{i} \in H^{\prime}$, we have that $\left|\left(x_{i}+K\right) \backslash K\right|<$ $\epsilon^{\prime}|K|$. Therefore

$$
\begin{aligned}
\left|\left(x_{i}+B\right) \cap K\right| & \geq\left|\left(x_{i}+B\right) \cap\left(x_{i}+K\right)\right|-\left|\left(x_{i}+K\right) \backslash K\right| \\
& >|B \cap K|-\epsilon^{\prime}|K| \\
& \geq(\alpha+\gamma)|K|-\gamma|K| \\
& =\alpha|K| .
\end{aligned}
$$

For the rest of this section we will assume we have a density function $d$ as guaranteed by Lemma 3.1 .

The next few lemmas are based on very similar results from 2. Recall that, given a commutative semigroup $(S,+), A \subseteq S$ and $k \in \mathbb{N}, k A=A+A+\cdots+A(k$ times) and $k \cdot A=\{k \cdot s: s \in A\}$.

Lemma 3.3. Let $(G,+)$ be a commutative group and let $A$ be a piecewise syndetic subset of $G$. Then $A-A$ is syndetic in $G$.

Proof. Pick $H \in \mathcal{P}_{f}(G)$ such that

$$
\left(\forall F \in \mathcal{P}_{f}(G)\right)(\exists y \in G)\left(F+y \subseteq \bigcup_{t \in H}(-t+A)\right) .
$$

Let $L=H-H$. We claim that $G \subseteq \bigcup_{s \in L}(-s+(A-A))$. To see this, let $x \in G$. Pick $y \in G$ such that $\{0, x\}+y \subseteq \bigcup_{t \in H}(-t+A)$. Pick $t_{1}$ and $t_{2}$ in $H$ such that $t_{1}+x+y \in A$ and $t_{2}+0+y \in A$. Then $x+\left(t_{1}-t_{2}\right) \in A-A$.

Lemma 3.4. Let $(G,+)$ be a commutative group and let $S \subseteq G$ such that $0 \in S$, $S=-S$, and $d(S)>0$. Then there is a subgroup $E$ of $G$ such that if $l \geq 2 / d(S)$, then $l S=E$.

Proof. We will show that there is some $j \leq 1 / d(S)$ such that $(2 j+1) S=(2 j) S$, and so $(k+1) S=k S$ for $k \geq 2 / d(S)$. Once we have shown this, let $k=\lceil 2 / d(S)\rceil$ and let $E=k S$. We have that $E+E=k S+k S=(2 k) S=E$. Since $S$ is symmetric, we also have that $E=-E$, so $E$ is closed under addition and the taking of inverses, hence, is a subgroup of $G$ as required.

Suppose instead that for each $j \leq 1 / d(S),(2 j) S \subsetneq(2 j+1) S$. We claim that, for each such $j,(2 j+1) S$ contains $j+1$ disjoint translates of $S$. This is a contradiction for $j=\lfloor 1 / d(S)\rfloor$.

The claim is true for $j=0$. For $j>0$, choose $x \in(2 j+1) S \backslash(2 j) S$. Then $x=s_{1}+\cdots+s_{2 j+1}$ with $s_{i} \in S$ for each $i$. We have

$$
\begin{array}{r}
S+x-s_{1}=S+s_{2}+\cdots+s_{2 j+1} \subseteq(2 j+1) S \\
\text { and }(2 j-1) S-s_{1} \subseteq(2 j) S \subseteq(2 j+1) S .
\end{array}
$$

Since $(2 j-1) S-s_{1}$ contains $j$ disjoint translates of $S$, it suffices to show that $S+x-s_{1}$ and $(2 j-1) S-s_{1}$ are disjoint. But if they intersect, then $t_{0}+x-s_{1}=$ $t_{1}+\cdots+t_{2 j-1}-s_{1}$ for some $t_{i} \in S$, from which it follows that $x=t_{1}+\cdots+t_{2 j-1}-t_{0} \in$ $(2 j) S$, contradicting the choice of $x$.

Lemma 3.5. Let $(G,+)$ be a commutative group and let $S \subseteq G$ such that $0 \in S$ and $d(S)>0$. Then there exists $Y \subseteq G$ such that for $k \geq 2 / d(S)$, we have $S-k S=Y$.

Proof. We suppose to the contrary that $S-(2 j) S \subsetneq S-(2 j+1) S$ for all $j \leq 1 / d(S)$ and show that $S-(2 j+1) S$ contains $j+1$ disjoint translates of $S$ for each such $j$, which is impossible for $j=\lfloor 1 / d(S)\rfloor$. 
The claim is true for $j=0$. For $j>0$, choose $x \in(S-(2 j+1) S) \backslash(S-(2 j) S)$. Then $x=s_{0}-s_{1}-\cdots-s_{2 j+1}$ with $s_{i} \in S$ for each $i$. We have

$$
\begin{gathered}
S+x-s_{0} \subseteq S-(2 j+1) S, \text { and } \\
S-(2 j-1) S-s_{0} \subseteq S-(2 j) S \subseteq S-(2 j+1) S,
\end{gathered}
$$

hence it suffices to show that $S+x-s_{0}$ and $S-(2 j-1) S-s_{0}$ are disjoint. But if they intersect then $t_{0}+x-s_{0}=t_{1}-t_{2}-\cdots-t_{2 j}-s_{0}$ for some $t_{i} \in S$, whence $x=t_{1}-t_{2}-\cdots-t_{2 j}-t_{0} \in S-(2 j) S$, contradicting the choice of $x$.

Lemma 3.6. Let $(G,+)$ be a commutative group and let $A \subseteq G$ such that $d(A)>0$. Assume that $E$ is a subgroup of $G$ such that if $l \geq 2 / d(A-A)$, then $l(A-A)=E$. If $k \geq 2 / d(A)$, then $(A-k A)=(A-k A)+E$.

Proof. Let $k=\lceil 2 / d(A)\rceil$ and let $X=A-k A$. For any $a \in A, 0 \in A-a$ and $d(A-a)=d(A)$, so by Lemma 3.5 if $Y=(A-a)-k(A-a)$ then also $Y=(A-a)-(k+1)(A-a)$ and so $X=X-(A-a)$. Letting $a$ range over the whole of $A$ gives $X=X+(A-A)$. Then for all $l \in \mathbb{N}, X=X+l(A-A)$, hence $X=X+E$ as required.

The following is our promised generalisation of Lemma 1.4.

Lemma 3.7. Let $(G,+)$ be a commutative group and assume that $n \cdot G$ is a central* set for each $n \in \mathbb{N}$. Let $A$ be a central subset of $G$. Then $d(A)>0$ and there exists $m \in \mathbb{N}$ such that if $k \geq 2 / d(A)$, then $m \cdot G \subseteq A-k A$.

Proof. Since $A$ is piecewise syndetic, by Lemma $3.1 d(A)>0$. Let $S=A-A$. Pick by Lemma 3.4 a subgroup $E$ of $G$ such that if $l \geq 2 / d(S)$, then $l S=E$. By Lemma 3.6 we have that if $k \geq 2 / d(A)$, then $(A-k A)=(A-k A)+E$.

Now $A-A$ is syndetic by Lemma [3.3 Let $l=\lceil 2 / d(A-A)\rceil$. Then $A-A \subseteq$ $l(A-A)=E$, so $E$ is syndetic. Pick $n \in \mathbb{N}$ and $x_{1}, x_{2}, \ldots, x_{n} \in G$ such that $G=\bigcup_{i=1}^{n}\left(-x_{i}+E\right)$. For $i \in\{1,2, \ldots, n\}$ pick $a_{i}<b_{i}$ in $\mathbb{N}$ and $j \in\{1,2, \ldots, n\}$ such that $a_{i} \cdot x_{i} \in\left(-x_{j}+E\right)$ and $b_{i} \cdot x_{i} \in\left(-x_{j}+E\right)$. Then $\left(b_{i}-a_{i}\right) \cdot x_{i} \in E-E=E$. Let $m=\prod_{i=1}^{n}\left(b_{i}-a_{i}\right)$. Then for each $i \in\{1,2, \ldots, n\}, m \cdot x_{i} \in E$.

We claim that $m \cdot G \subseteq E$. So let $x \in G$ and pick $i \in\{1,2, \ldots, n\}$ such that $x \in\left(-x_{i}+E\right)$. Then $x_{i}+x \in E$ so $m \cdot x_{i}+m \cdot x \in E$. Since $m \cdot x_{i} \in E$, we have that $m \cdot x \in E$.

Finally, let $k \geq 2 / d(A)$. Then $(A-k A)=(A-k A)+E$. Since $m \cdot G$ is central $^{*}, A \cap m \cdot G \neq \emptyset$. If $x \in A \cap m \cdot G$, then $x-k \cdot x \in(A-k A) \cap E$ so $E=(x-k \cdot x)+E \subseteq A-k A$. Thus $m \cdot G \subseteq A-k A$.

Proof of Lemma 1.4. Let $C$ be a central subset of $\mathbb{N}$. By [11, Exercise 4.3.8] $K(\beta \mathbb{Z})=K(\beta \mathbb{N}) \cup-K(\beta \mathbb{N})$. Therefore $C$ is a central subset of $\mathbb{Z}$. Given $n \in \mathbb{N}$, by [11, Lemma 6.6], $n \cdot \mathbb{N}$ is a member of every idempotent in $\beta \mathbb{N}$, so in particular is a member of every idempotent in $K(\beta \mathbb{N})$. Again using the fact that $K(\beta \mathbb{Z})=K(\beta \mathbb{N}) \cup-K(\beta \mathbb{N})$, we then have that $n \cdot \mathbb{Z}$ is central* in $\mathbb{Z}$. Therefore Lemma 3.7 applies.

We are now ready for our strengthening of Theorem 2.1

Corollary 3.8. Let $A$ be the matrix of Theorem 2.1. For any central subset $C$ of $\mathbb{N}$, there exist $\vec{x}$ and $\vec{y}$ in $C^{\omega}$ such that $\left(\begin{array}{ll}A & -I\end{array}\right)\left(\begin{array}{l}\vec{x} \\ \vec{y}\end{array}\right)=\overrightarrow{0}$ and all entries of $\left(\begin{array}{l}\vec{x} \\ \vec{y}\end{array}\right)$ are distinct. 
Proof. Let $C$ be a central set and pick an idempotent $p \in K(\beta \mathbb{N})$ such that $C \in p$. Let $C^{\star}=\{x \in C:-x+C \in p\}$. By [11, Lemma 4.14], if $x \in C^{\star}$, then $-x+C^{\star} \in p$. By Lemma 3.7 pick $m \in \mathbb{N}$ such that for all $k \geq 2 / d\left(C^{\star}\right), m \cdot \mathbb{Z} \subseteq C^{\star}-k C^{\star}$. Pick $M \in \mathbb{N}$ such that $2^{M+1} \geq m-2+\left\lceil 2 / d\left(C^{\star}\right)\right\rceil$.

Let $P$ be the $(M+1) \times 2^{M+1}$ matrix consisting of rows $0,1, \ldots, M$ and columns $0,1, \ldots, 2^{M+1}-1$ of $A$ and let $I_{M+1}$ be the $(M+1) \times(M+1)$ identity matrix. If the columns of $\left(\begin{array}{c}I_{M+1} \\ P\end{array}\right)$ are reversed, then the first nonzero entry in each column is 1 , so by [11, Corollary 15.6], $\left(\begin{array}{c}I_{M+1} \\ P\end{array}\right)$ is image partition regular over $\mathbb{N}$ and thus by [11, Theorem $15.24(\mathrm{~m})]$ we may choose $\vec{x}=\left\langle x_{i}\right\rangle_{i=0}^{2^{M+1}-1}$ and $\vec{y}=\left\langle y_{i}\right\rangle_{i=0}^{M}$ in $C^{\star}$ such that all entries of $\left(\begin{array}{l}\vec{x} \\ \vec{y}\end{array}\right)$ are distinct.

Now let $r \geq M$ and assume that we have chosen $\left\langle x_{i}\right\rangle_{i=0}^{r+1}-1$ and $\left\langle y_{i}\right\rangle_{i=0}^{r}$ in $C^{\star}$ such that for each $n \in\{0,1, \ldots, r\}, y_{n}=2 x_{n}+\sum_{j=2^{n}}^{2^{n+1}-1} x_{n}$ and all of the $x_{i}$ and $y_{j}$ are distinct. For $\left.2^{r+1} \leq t \leq 2^{r+2}-1\right\}$, let $z_{t}=x_{r+1}$. Let $k=2^{r+1}-m+2$. Then $k \geq\left\lceil 2 / d\left(C^{\star}\right)\right\rceil$ and $2 x_{r+1}+\sum_{t=2^{r+1}}^{2^{r+1}+m} z_{t}=m \cdot x_{r+1} \in m \cdot \mathbb{Z}$ so by Lemma 3.7 we may pick $v \in C^{\star}$ and pick $z_{t} \in C^{\star}$ for $\left.2^{r+1}+m-2 \leq t \leq 2^{r+2}-1\right\}$ such that $v=2 x_{r+1}+\sum_{t=2^{r+1}}^{2^{r+2}-1} z_{t}$. Let

$$
D=\left(-v+C^{\star}\right) \cap \bigcap_{t=2^{r+1}}^{2^{r+2}-1}\left(-z_{t}+C^{\star}\right) .
$$

Then $D \in p$, which is an idempotent, so by [11, Theorem 5.8] pick a sequence $\left\langle w_{n}\right\rangle_{n=1}^{\infty}$ in $\mathbb{N}$ such that $F S\left(\left\langle w_{n}\right\rangle_{n=1}^{\infty}\right)=\left\{\sum_{t \in F} x_{t}: F \in \mathcal{P}_{f}(\mathbb{N})\right\} \subseteq D$. We may assume that the sequence $\left\langle w_{n}\right\rangle_{n=1}^{\infty}$ is increasing. Choose $n\left(2^{r+1}\right) \in \mathbb{N}$ such that $w_{n\left(2^{r+1}\right)}>\max \left(\left\{x_{i}: i \in\left\{0,1, \ldots, 2^{r+1}-1\right\}\right\} \cup\left\{y_{i}: i \in\{0,1, \ldots, r\}\right\}\right)$. Given $j \in\left\{2^{r+1}, 2^{r+1}+1, \ldots, 2^{r+1}-2\right\}$, having chosen $n(j)$, pick $n(j+1)>n(j)$ such that $w_{n(j+1)}>z_{j}+w_{n(j)}$.

For $2^{r+1} \leq j \leq 2^{r+1}-1$, let $x_{j}=z_{j}+w_{n(j)}$ and let $y_{r+1}=2 x_{r+1}+\sum_{j=2^{r+1}}^{2^{r+2}-1} x_{j}$. Then each $x_{j} \in C^{\star}$ and $y_{r+1}=v+\sum_{j=2^{r+1}}^{2^{r+2}-1} w_{n(j)} \in C^{\star}$. Finally,

$$
\begin{aligned}
\max & \left(\left\{x_{i}: i \in\left\{0,1, \ldots, 2^{r+1}-1\right\}\right\} \cup\left\{y_{i}: i \in\{0,1, \ldots, r\}\right\}\right) \\
& <x_{2^{r+1}}<x_{2^{r+1}+1}<\cdots<x_{2^{r+2}-1}<y_{r+1} .
\end{aligned}
$$

Remark 3.9. We remark that Corollary 3.8 holds more generally. Let $G$ be a commutative group with the property that, for every $n \in \mathbb{N}, n \cdot G$ is a central subset of $G$. Then Corollary 3.8 holds with $\mathbb{N}$ replaced by $G$. The proof is essentially the same.

\section{RefERENCES}

[1] L. N. Argabright and C. O. Wilde, Semigroups satisfying a strong Følner condition, Proc. Amer. Math. Soc. 18 (1967), 587-591. MR0210797 (35 \#1683)

[2] Ben Barber, Neil Hindman, and Imre Leader, Partition regularity in the rationals, J. Combin. Theory Ser. A 120 (2013), no. 7, 1590-1599, DOI 10.1016/j.jcta.2013.05.011. MR3092686

[3] H. G. Dales, A. T.-M. Lau, and D. Strauss, Banach algebras on semigroups and on their compactifications, Mem. Amer. Math. Soc. 205 (2010), no. 966, vi+165, DOI 10.1090/S00659266-10-00595-8. MR2650729(2011d:43001) 
[4] H. Furstenberg, Recurrence in ergodic theory and combinatorial number theory, Princeton University Press, Princeton, N.J., 1981. M. B. Porter Lectures. MR603625 (82j:28010)

[5] Neil Hindman, Finite sums from sequences within cells of a partition of $N$, J. Combinatorial Theory Ser. A 17 (1974), 1-11. MR0349574 (50 \#2067)

[6] Neil Hindman, Imre Leader, and Dona Strauss, Infinite partition regular matrices: solutions in central sets, Trans. Amer. Math. Soc. 355 (2003), no. 3, 1213-1235, DOI 10.1090/S00029947-02-03191-4. MR1938754 (2003h:05187)

[7] Neil Hindman, Imre Leader, and Dona Strauss, Open problems in partition regularity, Combin. Probab. Comput. 12 (2003), no. 5-6, 571-583, DOI 10.1017/S0963548303005716. Special issue on Ramsey theory. MR2037071 (2005e:05147)

[8] Neil Hindman and Dona Strauss, Density in arbitrary semigroups, Semigroup Forum 73 (2006), no. 2, 273-300, DOI 10.1007/s00233-006-0622-5. MR2280825 (2009b:11019)

[9] Neil Hindman and Dona Strauss, Sets satisfying the central sets theorem, Semigroup Forum 79 (2009), no. 3, 480-506, DOI 10.1007/s00233-009-9179-4. MR2564059 (2011m:22005)

[10] Neil Hindman and Dona Strauss, Density and invariant means in left amenable semigroups, Topology Appl. 156 (2009), no. 16, 2614-2628, DOI 10.1016/j.topol.2009.04.016. MR2561213 (2011b:43003)

[11] Neil Hindman and Dona Strauss, Algebra in the Stone-Čech compactification, de Gruyter Textbook, Walter de Gruyter \& Co., Berlin, 2012. Theory and applications; Second revised and extended edition [of MR1642231]. MR2893605

[12] Keith R. Milliken, Ramsey's theorem with sums or unions, J. Combinatorial Theory Ser. A 18 (1975), 276-290. MR0373906 (51 \#10106)

[13] Richard Rado, Studien zur Kombinatorik (German), Math. Z. 36 (1933), no. 1, 424-470, DOI 10.1007/BF01188632. MR1545354

[14] I. Schur, Über die Kongruenz $x^{m}+y^{m}=z^{m} \quad(\bmod p)$, Jahresbericht der Deutschen Math.Verein. 25 (1916), 114-117.

[15] Alan D. Taylor, A canonical partition relation for finite subsets of $\omega$, J. Combinatorial Theory Ser. A 21 (1976), no. 2, 137-146. MR0424571 (54 \#12530)

[16] B. van der Waerden, Beweis einer Baudetschen Vermutung, Nieuw Arch. Wiskunde 19 (1927), 212-216.

School of Mathematics, University of Birmingham, Edgbaston, Birmingham, B15 2TT, UNITED Kingdom

E-mail address: b.a.barber@bham.ac.uk

Department of Mathematics, Howard University, Washington, DC 20059

E-mail address: nhindman@aol.com

Department of Pure Mathematics and Mathematical Statistics, Centre for Mathematical Sciences, Wilberforce Road, Cambridge, CB3 0WB, United Kingdom

E-mail address: i.leader@dpmms.cam.ac.uk

Department of Pure Mathematics, University of Leeds, Leeds LS2 9J2, United KingDOM

E-mail address: d.strauss@hull.ac.uk 